\title{
Agitation of non-Brownian particles by active matrix of kinesin- microtubule motor proteins
}

\author{
Keisuke Meguriya ${ }^{1}$, Tsuyoshi Yokoyama $^{1}$, Naritaka Kobayashi ${ }^{2,3}$, Hiroshi Y. \\ Yoshikawa ${ }^{1,2,4}$, Seiichiro Nakabayashi ${ }^{1,2}$ and Ryuzo Kawamura ${ }^{1,2}$ \\ ${ }^{1}$ Department of Chemistry, Saitama University, Saitama 338-8570, Japan \\ ${ }^{2}$ Division of Strategic Research and Development, Graduate School of Science and Engineering, \\ Saitama University, Saitama 338-8570, Japan \\ ${ }^{3}$ Department of Electronic Systems Engineering, School of Engineering, The University of Shiga \\ Prefecture, Hassaka Cho 2500, Shiga 522-8533, Japan \\ ${ }^{4}$ Graduate School of Engineering, Osaka University, Suita, Osaka 565-0871, Japan
}

E-mail: ryuzo@mail.saitama-u.ac.jp

\begin{abstract}
In living organisms, many dynamic mechanisms are driven by motor proteins on a wide scale for tasks including the assembly of hierarchical structures at the nano to micrometre scales and macroscopic movements with hierarchical structures. Such complicated assemblies and sophisticated functions are intriguing for applications in nano and microengineering. Using motor proteins may enable multimolecular assembly in artificial systems by reproducing simple molecular movements using established methods such as motility assays of kinesin and microtubules. However, building a multimolecular system and selecting the target functions are key points to consider for potential applications. We use an active matrix consisting of crosslinked microtubules driven by kinesin to agitate microscopic objects that are not moved by thermal fluctuation, that is, non-Brownian particles. This method may contribute to enhance various self-assembly processes for larger objects. The resulting isotropic agitating properties are compared with those of other agitation methods based on external forces exerted by electric motors. The active matrix may provide a new type of mesoscopic scale actuator to perform stochastic mechanical agitation.
\end{abstract}

Keywords: motor protein, active matrix, mechanical agitation, non-Brownian particles, self-assembly

\section{Introduction}

When assembling micro-components into a macroscopic structure, bottom-up approaches such as self-assembly or phase separation have been developed to replace pick-andplace handling of very small parts. In self-assembly, agitation and binding of the building blocks are the essential steps, ${ }^{1-3}$ and the control of these processes has been pursued to obtain assembly structures such as aggregation patterns and colloid crystals. Research is being increasingly focused on more complex (e.g. hierarchical) structures with fewer defects and larger-scale macroscopic structures. ${ }^{4,5}$ The geometries of building blocks, such as sticky domains and chemical components, have been the main concerns for applications such as Janus particles, patchy particles, and DNA origami. In addition, interactions of solvophobic surfaces, cyclodextrin for host-guest chemistry, protein-specific interactions, and DNA annealing are being harnessed. ${ }^{1,6-22}$

With the increasing complexity and scale in assembly designs, structural stability, morphological purity, production efficiency, and other problems have emerged, ${ }^{23-28}$ and the movements of building blocks are being carefully investigated because they affect the configurations that originate macroscopic structures. ${ }^{5,29,30}$ In conventional self-assembly, including phase separation, components are conveyed via Brownian motions. Numerous collisions of solvent molecules allow translocation and turn for changing the direction of the components, generating random trajectories. In addition to Brownian particles, larger components of approximately 
$100 \mathrm{~nm}-1 \mathrm{~mm}$ in diameter move less by thermal fluctuation and they sediment faster, tending to be kinetically arrested. ${ }^{1}$ To overcome kinetic arrest of non-Brownian particles, an additional driving force for agitation should be available during assembly. ${ }^{31,32}$

Various active agitation methods are available, from simple mechanical vibration, such as sonication, ${ }^{17,32-37}$ to the use of force fields, such as capillary forces under solvent evaporation, ${ }^{38,39}$ and centrifugal forces. ${ }^{40}$ Although these methods generate agitation, they drive the components unidirectionally, and the interparticle position is less affected by the force fields of the sweeping gas/liquid interface and centrifugal force or by the shock wave of sonication. In these methods, changes in the moving direction occur due to thermal fluctuation and collisions among the components if the particle density is sufficient. ${ }^{41-43}$ Thus, random trajectories with frequent turns are less drawn by thermal fluctuations for large non-Brownian particles. The resulting immobility may impede the formation of aligned structures such as lattices, ${ }^{16,44}$ in which the component orientation is important for correct binding.

Few methods that promote interparticle displacement are available. For instance, Janus magnetic particles which exhibit different motions according to the magnetic orientation of individual particles under an altering magnetic field can be used to induce reorientation and collision of particles. ${ }^{45-47}$ Alternatively, active materials driven by chemical reactions can be used. ${ }^{41,48-50}$ In principle, the particles move individually without outer fields, causing interparticle displacement. However, asymmetric modification of individual particles and specific chemical reactions are necessary, possibly limiting the design of arbitrary building blocks for complex and hierarchical self-assembly. In addition to examples in artificial systems, living cells perform elaborate and complicated dynamic assemblies. In particular, motor proteins enhance the cytoplasmic diffusion of molecules and organelles, that is, they perform agitation inside the cells by consuming chemical energy. ${ }^{51}$ Inspired by this mechanism, we use motor proteins for agitation of micro-objects.

Kinesin and microtubules constitute a linear motor of proteins. The kinesin motor walks along the path set by the microtubule in one direction consuming the chemical energy of available adenosine triphosphate (ATP). Hence, kinesin and microtubules have often been employed in the design of miniature devices to generate nano/micrometric movements controlling the driving directions of molecules. ${ }^{52-56} \mathrm{In}$ a typical setup, sliding motions of microtubules can be observed under a microscope driven by kinesin on a solid substrate. It has been shown that chemical crosslinking of microtubules to form a network allows generating fluctuating motions down to the submillimetre scale on a kinesin-decorated surface, despite the microtubule orientations being random. ${ }^{57,58}$ When living cells are suspended on this kinesin-driven active matrix of crosslinked microtubules, the suspended cells, which are originally adhesive, fluctuate and cell aggregation enhances. ${ }^{59}$ Therefore, the active matrix can agitate microscale objects to cause collisions, potentially contributing to various selfassembly methods. Nevertheless, the uniqueness of object conveyance remains unclear, particularly in terms of agitation. Thus, we characterised the stochastic driving of a kinesindriven active matrix considering interparticle displacements in this study. The agitation properties were evaluated through comparisons with simple agitation methods that use an external vibrating force and with passive conditions to observe particle motions on the $x y$ plane during sinking.

\section{Methods}

\subsection{Tubulin and kinesin purification}

Tubulin was purified from fresh porcine brain which was obtained from the slaughterhouse according to an established method that uses a high ionic strength buffer to remove the microtubule-associated proteins. ${ }^{60}$ The purified tubulins were aliquoted and flash-frozen in liquid nitrogen for storage at $-80{ }^{\circ} \mathrm{C}$ until use. Tubulin was maintained in a BRB80 buffer ( $80 \mathrm{mM}$ PIPES, $1 \mathrm{mM}$ EGTA, and $1 \mathrm{mM} \mathrm{MgCl}_{2}$ at $\mathrm{pH} 6.8$ by $\mathrm{KOH})$ unless modifications were noted. Kinesin was prepared as a fusion protein with green fluorescent protein (kinesinGFP) according to an established method with a modification to omit ion-exchange chromatography before microtubuleaffinity purification. ${ }^{61}$

\subsection{Preparation of crosslinked microtubules}

The crosslinked microtubules were prepared as reported in Refs. 57,58. The microtubules were polymerised from tubulins $(100 \mu \mathrm{M})$ in a polymerisation buffer $(80 \mathrm{mM}$ PIPES, $1 \mathrm{mM}$ EGTA, $6 \mathrm{mM} \mathrm{MgCl} 2,5 \mathrm{mM} \mathrm{GTP}, \mathrm{pH} \mathrm{6.8)}$ at $37^{\circ} \mathrm{C}$ for $30 \mathrm{~min}$.

For crosslinking, bis- $N$-hydroxysuccinimidyl ester polyethylene glycol (SUNBRIGHT DE-034GS, NOF, Tokyo, Japan) was added to the polymerised microtubules at a molar ratio of 2:1 (tubulin:CL), corresponding to an equimolar ratio of the NHS group to the tubulins (NHS/tubulin =1). The crosslinking reaction was performed by incubation at $37{ }^{\circ} \mathrm{C}$ for $1 \mathrm{~h}$. The crosslinked microtubules were depolymerised by chilling on ice for $30 \mathrm{~min}$ and polymerised again by incubating at $37^{\circ} \mathrm{C}$ for $30 \mathrm{~min}$ to obtain uniformly crosslinked microtubules. The crosslinked microtubules were diluted twentyfold in a motility assay buffer ( $80 \mathrm{mM}$ PIPES, $1 \mathrm{mM}$ EGTA, $1 \mathrm{mM} \mathrm{MgCl} 2,0.5 \mathrm{mg} / \mathrm{mL}$ casein, $1 \mathrm{mM} \mathrm{TCEP}$, and $10 \mu \mathrm{M}$ paclitaxel, $\mathrm{pH}$ 6.8). The microtubules in this solution were stabilised by paclitaxel, and this solution was kept at room temperature until perfusion to a kinesin-decorated chamber. 
(a)

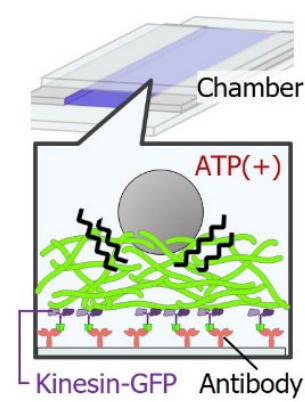

(c)

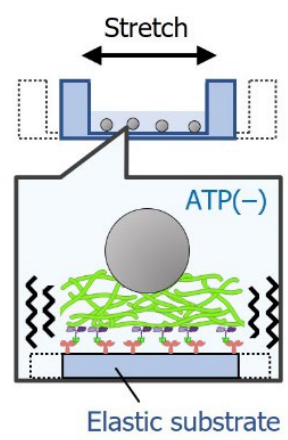

(b)

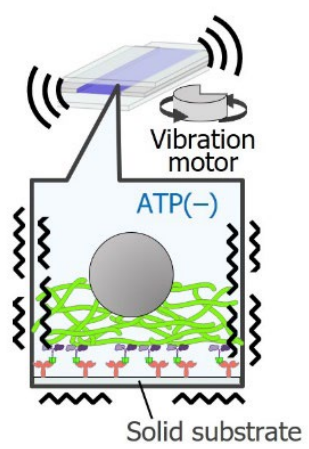

(d)

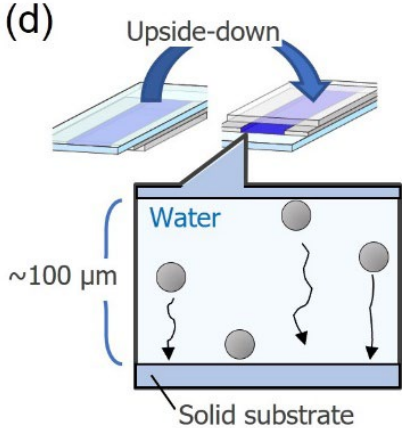

Figure 1. Schematic illustration of kinesin-driven active matrix of microtubule networks $(a)$. Setups using external forces of electric motor which vibrate solid chamber (b) or stretching of elastic substrate STREX ${ }^{\circledR}$ (c). (b) and (c) are decorated with matrices but cannot be activated without the addition of ATP. For comparison, a setup for observing free-falling microparticles was also prepared $(d)$. The chamber was turned upside down to start the process in which the particles sank to the bottom by an approximate height of $100 \mu \mathrm{m}$ in around $20 \mathrm{~s}$.

\subsection{Preparation of kinesin-driven active matrix}

The kinesin-driven active matrix was prepared based on a conventional motility assay protocol using crosslinked microtubules, as reported in Ref. 58 [Figure 1(a)]. As a sample chamber, a flow cell was used, and solutions containing each component were perfused step-by-step as follows [Figure 2]. The chamber was made by assembling a pair of cover glasses separated by spacers of Parafilm $\AA$ (Bemis Flexible Packaging, Neenah, WI) with approximate inner dimensions of $3 \times 18 \times 0.1 \mathrm{~mm}^{3}$ (width $\times$ length $\times$ height) and approximate volume of $5 \mu \mathrm{L}$. The chamber was filled with $0.2 \mathrm{mg} / \mathrm{mL}$ antiGFP antibody (A11122, Invitrogen, Carlsbad, CA) for $15 \mathrm{~min}$, followed by perfusion of $20 \mu \mathrm{L}$ casein solution (approximately $0.5 \mathrm{mg} / \mathrm{mL}$ in BRB80 buffer) for washing and blocking the remaining glass surface. After 5 min incubation with casein, $15 \mu \mathrm{L}$ of a 210 -nM GFP-fused kinesin solution ( $80 \mathrm{mM}$ PIPES, $1 \mathrm{mM}$ EGTA, $1 \mathrm{mM} \mathrm{MgCl} 2,0.5 \mathrm{mg} / \mathrm{mL}$ casein, $1 \mathrm{mM}$ TCEP, pH 6.8) was added and incubated for $10 \mathrm{~min}$ to bind to the anti-GFP antibody. The crosslinked microtubules diluted in the motility assay buffer were introduced and allowed to sit for more than $2 \mathrm{~h}$ to settle until starting the motility assay. The entrance and exit of the

chamber were covered with a polyvinylidene chloride film to avoid evaporation.

The assay started by perfusing $20 \mu \mathrm{L}$ of motility buffer containing $5 \mathrm{mM}$ ATP and suspended silica microparticles with a diameter of $5 \mu \mathrm{m}$ (Sicaster $\AA$ plain, 43-00-503, micromod Partikeltechnologie, Rostock, Germany). All solutions in the chamber were exchanged by pulling with a syringe pump (YSP-202, YMC, Kyoto, Japan) at a flow rate of $10 \mu \mathrm{L} / \mathrm{min}$, which was optimised to maintain reproducibility of the particle movements on the active matrix (see Figures S1-S3).

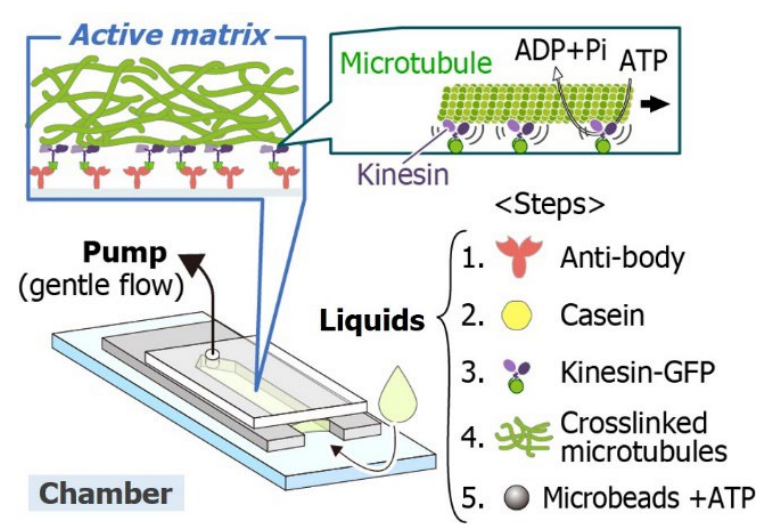

Figure 2. Preparation of active matrix in a flow-cell chamber. Sample liquids containing the building blocks of each molecule were added step-by-step with gentle flow control by an automatic pump $(10 \mu \mathrm{L} / \mathrm{min}$ for entrance size of approximately $3 \times 0.1 \mathrm{~mm}$ (width $\times$ height). (ADP, adenosine diphosphate; Pi, phosphate)

\subsection{Electric motor vibration and cyclic stretching of elastic chamber}

To evaluate agitation of silica particles suspended in matrices of crosslinked microtubules on kinesin without the addition of ATP, other vibration methods were tested.

One method was vibrating the entire flow cell using a coinshaped vibrating electric motor $(13,000 \mathrm{rpm}, 1.8 \mathrm{G}$ shock; FM34F, Tokyo Parts Industrial, Tokyo, Japan) [Figure 1(b)]. This motor was attached to a microscope stage using doublesided tape. The horizontal vibration was transmitted to the particles through the stage and sample holder, which was also fixed to the flow cell with double-sided tape [Figure 3(a)]. The practical vibration of the chamber was measured to be reciprocal with a $0.5 \mu \mathrm{m}$ stroke at a frequency of $180 \mathrm{~Hz}$ [Figures 3(b-d)].

Another method was using elastic substrate Strex ${ }^{\circledR}$ (STB-CH04, Strex, Osaka, Japan) designed for cell culture. The elastic substrate was stretched using an inhouse stretching device consisting of a linear actuator (PQ12, Actuonix Motion Devices, BC, Canada) controlled by an Arduino board (Uno R3, Arduino, Zug, Switzerland) [Figures 1(c) and 4]. The stretchable-membrane bottom of the Strex ${ }^{\circledR}$ chamber 
$(20 \times 20 \mathrm{~mm})$ was subjected to cyclic stretching at a frequency of $0.5 \mathrm{~Hz}$ and set to produce a $10 \%$ linear strain, that is, a $2 \mathrm{~mm}$ stroke. The silica microparticles suspended in water were introduced into the chamber, and the movements were observed by microscopy.

\subsection{Microscopic imaging of silica microparticles and analysis}

The motions of the silica microparticles were captured using an sCMOS camera (Zyla-4.2P-CL10, Andor, Belfast, Ireland) attached to a microscope (Ti2-E, Nikon, Tokyo, Japan). An objective lens of 20×/ NA 0.45 ELWD (Nikon) was used for bright-field imaging. Sequential images were
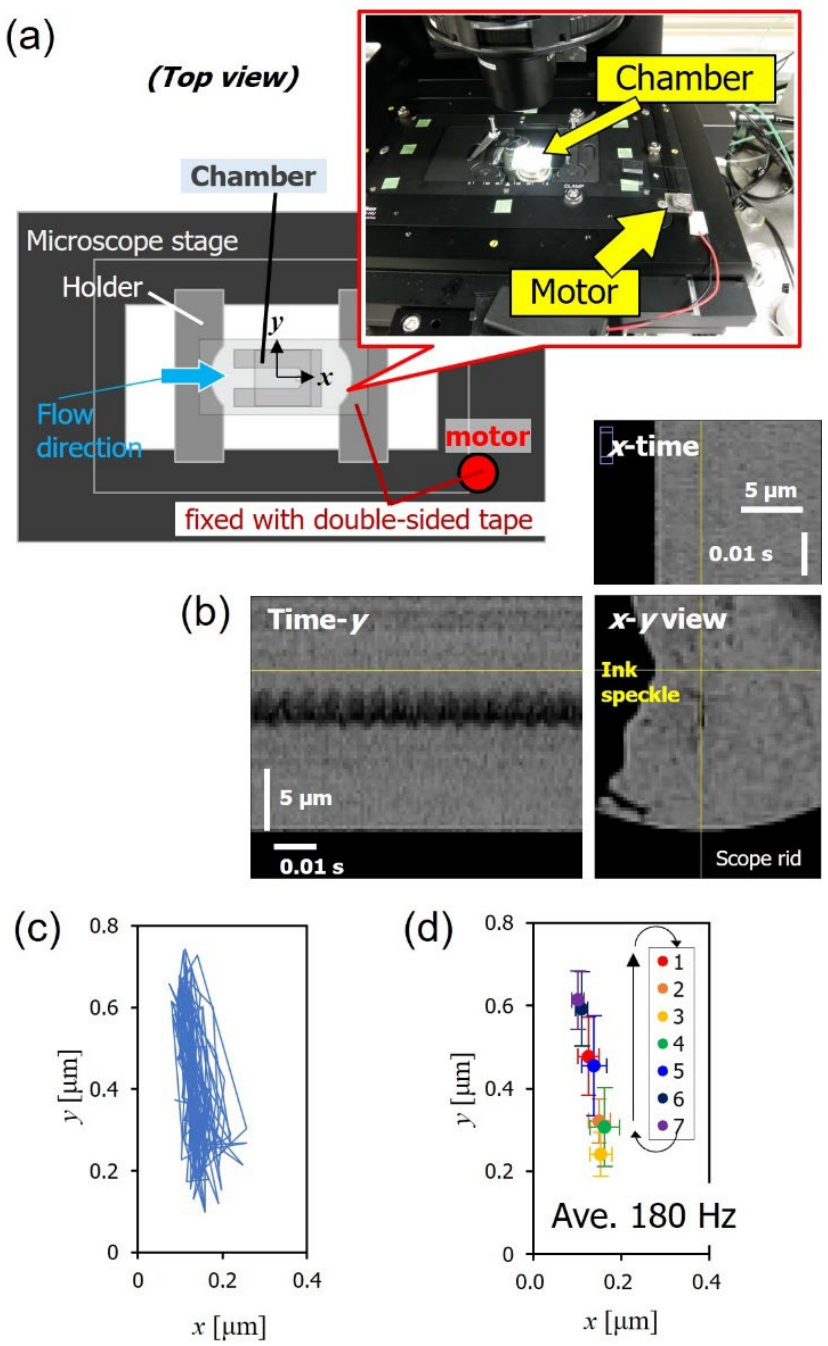

Figure 3. Chamber setup with electric vibration motor (a). The actual vibration of the chamber was observed using a high-speed camera of $1200 \mathrm{frame} / \mathrm{s}(\mathrm{b})$. The vibrating pattern of the ink speckle marked on the glass substrate was used to obtain the trajectory caused by vibration from the images $(c)$. The trajectory was divided into seven periods to obtain the average path $(d)$. The almost reciprocal vibration obtained from an approximate travel of $0.5 \mu \mathrm{m}$ was determined at a frequency of $180 \mathrm{~Hz}$. obtained for at least $1000 \mathrm{~s}$ at intervals of $5 \mathrm{~s}$ unless otherwise stated. Image analysis was conducted using the Image-Pro Analyzer 7.0 software (Japan Media Cybernetics, Tokyo, Japan). The movements of the particles were automatically tracked using the software with manual corrections for some misrecognition errors. The mean squared displacements (MSDs) of the trajectories and fluctuations of interparticle distances $[d d x$, $d d y$ ] were calculated from positions $(x, y)$ at each time step over $1000 \mathrm{~s}$.

\section{Results and discussion}

\subsection{Particle motion on kinesin-driven active matrix and comparison with other agitation methods}

An active matrix of crosslinked microtubules driven by kinesin was prepared based on an existing method, ${ }^{58}$ and sample preparation was improved by automating liquid handling to increase the uniformity and reproducibility of moving micro-objects (see Figures S1-S3). As a microtubule is a relatively rigid and brittle fibre, slow liquid flow was key to prevent defects in crosslinked microtubules owing to shear stress. To analyse particle motion, silica microparticles (5 $\mu \mathrm{m}$ in diameter) were dispersed to buffer in the flow cell [Figure 1(a)], and the trajectories were traced using the recorded movie data. Silica was the material selected to enhance the contact to the bottom substrate because it has a density of $2.2 \mathrm{~g} / \mathrm{cm}^{3}$ for easy sinking, unlike a resin such as polystyrene, which has a density comparable to that of water and thus sinks very slowly.

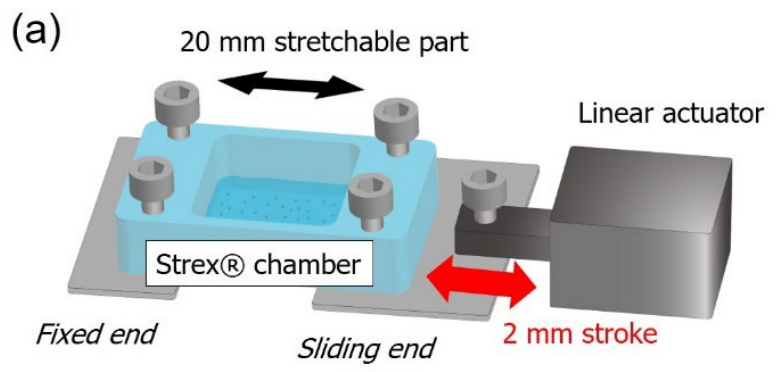

(b)

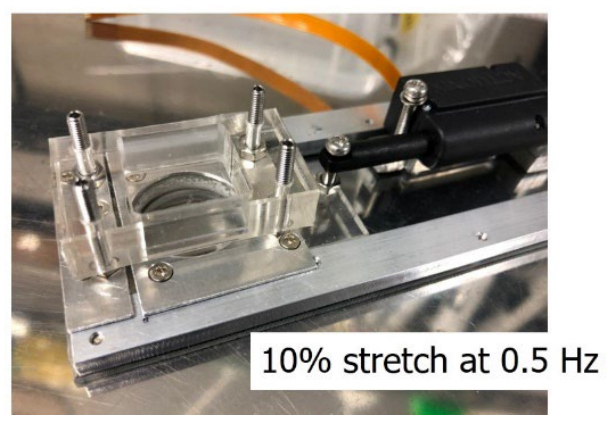


Active matrix

(a)

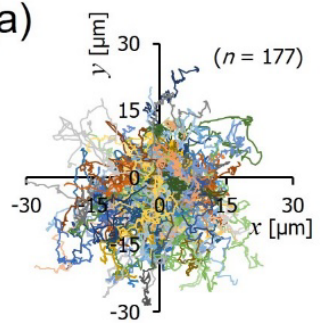

(e)

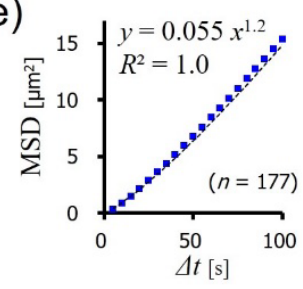

Vibration motor

(b)

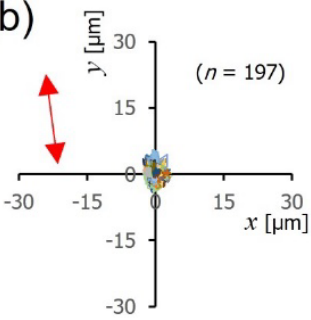

(f)

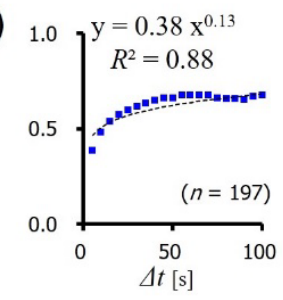

The particles in the active matrix showed random trajectories [Figure 5(a)]. The MSD shows an almost normal diffusion [Figure 5(e)], but it was slightly super-diffusive because $\alpha$ was larger than 1 in relation to the MSD proportional to $\Delta t^{\alpha}$ according to Ref. 58. Super-diffusion was caused by particles sometimes showing straight motion owing to the occasional release of elastic energy accumulated in the deformed substrate of the microtubules by the force from kinesin.

For comparison, other methods which use external forces were evaluated regarding the fluctuation of particles suspended in the matrix without ATP to keep them passive. The particle trajectories in the flow cells under vibration with an electric motor [Figures 1(b) and 3] and in a cyclically stretched elastic chamber [Figures 1(c) and 4] are shown in Figures 5(b) and 5(c), respectively. The particles in the vibrating chamber and some particles in the stretching chamber showed very low mobility and subtle one-directional trends biased to the external force directions, as indicated by the red arrows in Figures 5(b) and 5(c). The MSDs showed the effects of the electric motor vibration and stretching substrate [Figures 5(f) and 5(g)], which resulted in trajectories with subdiffusion, as parameter $\alpha$ was clearly below 1 . The small oscillations shown in Figure 5(g) were due to the gap between the image sampling and stretching cycle. As the matrices of crosslinked microtubules can trap and immobilise the microparticles, a control case with the microparticles suspended in water instead of the matrices was evaluated [Figures S4(a) and S4(b)]. The particles suspended in more fluidic conditions showed larger movements and longer trajectories in the direction of motor vibration, and the MSD showed super-diffusion $(\alpha=1.8)$ [Figures S4(b) and S4(c)]. The straight travel was mainly attributed to the inertia and
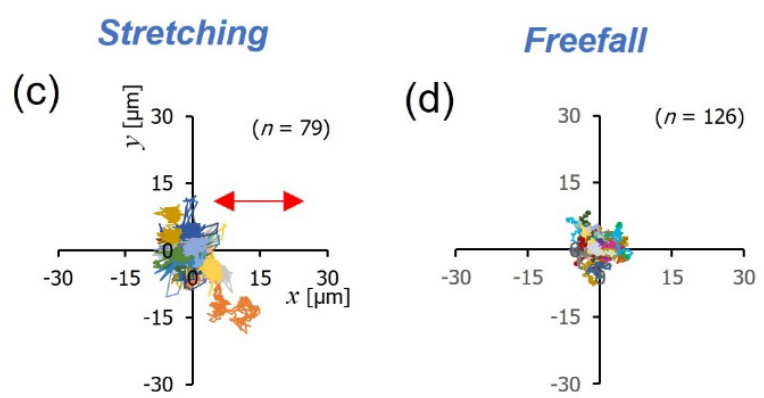

(g)

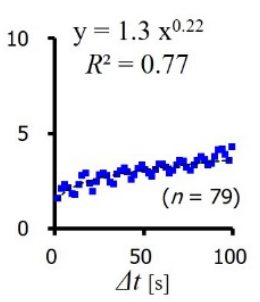

fluidity of the water suspension, in which the particles can be vibrated in the open chamber but are affected by the vibration directions [Figures S1(b)-(f)]. On the other hand, the particles do not move when the chamber is closed and small enough. The particles in the stretching chamber without the matrix did not move, and few cases $(N=3)$ showed much longer travel above $100 \mu \mathrm{m}$, which can be attributed to the flow of liquid caused by macroscopic chamber deformations [Figure S4(f)]. The MSD of the particles with restricted mobility resulted in sub-diffusion, as parameter $\alpha(=0.6)$ was clearly less than 1 [Figure $\mathrm{S} 4(\mathrm{~g})$ ].

The random fluctuation of microparticles by collision with water molecules was evaluated through free falling. The movements of the particles on the horizontal $x y$ plane were traced during sinking after turning the chamber upside down [Figure 1(d)]. The particles were suspended in water, and the glass substrate was coated with casein to prevent particle sticking; otherwise, the particles were not at all along the $z$ axis direction in the matrix of crosslinked microtubules. Figure 5(d) shows random and isotropic trajectories similar to those obtained from the active matrix [Figure 5(a)]. However, the travel was limited owing to the fixed chamber depth. The MSD was not calculated because of the short observation time.

To clarify the trends in the trajectories shown in Figures $5(a)-5(d)$ over time, the probability distributions of the displacements along the $x$ and $y$ axes were determined, obtaining the results shown in Figure 6. The kinesin-driven active matrix provided nearly Gaussian distributions [Figure 6(a)], and the bell shapes became wider over a longer period up to $50 \mathrm{~s}$ along both the $x$ and $y$ axes. The mean displacements were zero, and the bell shapes were symmetric with respect to the centre, indicating random movements (e.g. Brownian motions) without notable offset. Figure 5(e) shows the relation 
between the period and width $\sigma$ obtained by Gaussian fitting. With the largest time window of $500 \mathrm{~s}$, the probability distribution became almost flat, and fitting was not possible.

The same analysis was applied the other agitation methods of electric motor vibration, stretching, and free falling [Figures 5(b), 5(c), and 5(d), respectively]. For electric motor vibration, the width of the Gaussian distribution remained constant for the various periods, as shown in Figures 6(b) and 6(f). The stretching of the elastic substrate showed a slight effect on spreading the probability distribution according to the period along both the $x$ and $y$ axes, but with much less intensity than in the active matrix [Figures $6(\mathrm{c})$ and $6(\mathrm{~g})$ ]. For free fall, the probability distribution spread isotropically over the periods, being similar to the active matrix [Figures $6(\mathrm{~d})$ and $6(\mathrm{~h})$ ], but the period was limited by the total vertical travel. Thus, the active matrix provided the highest dispersion of microparticles isotropically and over long distances.

The variations in the distances between two particles were evaluated using parameters $\overline{d d x}$ and $\overline{d d y}$ as shown in Figure 7(a). For paired combinations of 10 tracked particles (combinations of ${ }_{10} \mathrm{C}_{2}=45$ ), the average variations along the $x$ - and $y$-axis components of the relative positions for the observation time were calculated [Figures 7(b)-7(d)]. The fluctuations in the interparticle distance on the active matrix showed a nearly isotropic distribution over an area with an approximate radius of $0.4 \mu \mathrm{m}$ during the $5 \mathrm{~s}$ intervals. In contrast, those on the vibration motor [Figure 7(c)] and stretching chamber [Figure 7(d)] were mostly less than $0.1 \mu \mathrm{m}$ regardless of the force directions. When the microparticles were suspended in water, the interparticle distance varied along the fluctuating directions parallel to the external forces but did not spread isotropically, and the displacements were limited [Figures S4(d) and S4(h)]. Thus, only the kinesindriven active matrix enhanced the interparticle fluctuation in two dimensions, possibly leading to isotropic collisions among the particles.

\subsection{Discussion}

Owing to their small size and directed drive, motor proteins are often employed in the development of nanotools which perform oriented micromovements. In this study, a random driving force from a non-oriented multimolecular system was characterised aiming to confirm stochastic agitation. Through the crosslinked network of microtubules, multiple driving forces of kinesin were successfully transmitted with some additivity to the movement of silica particles $(5 \mu \mathrm{m}$ in diameter) for displacements over several tens of micrometres,

\section{Active matrix}

(a)
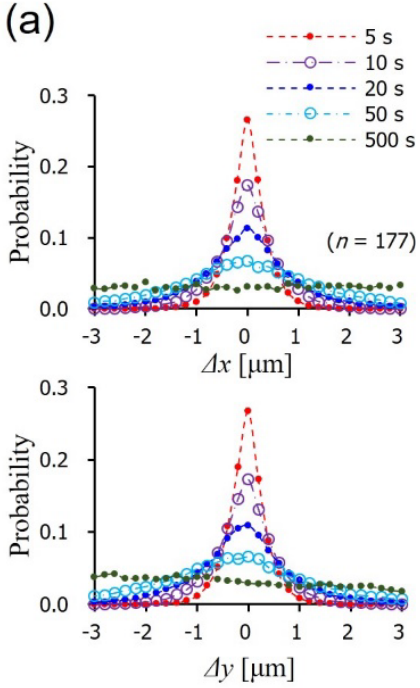

(e)

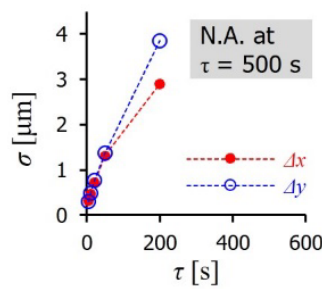

Vibration motor

(b)
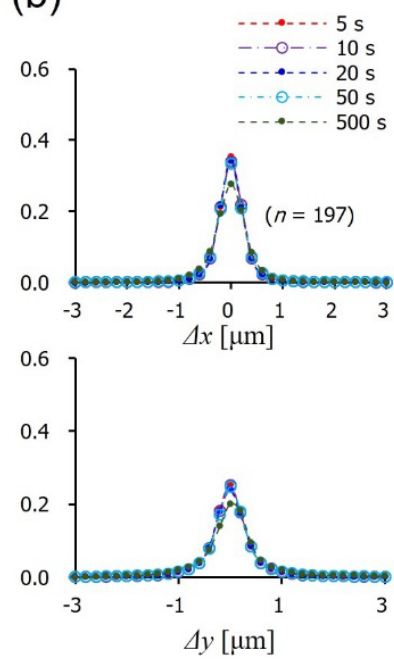

(f)

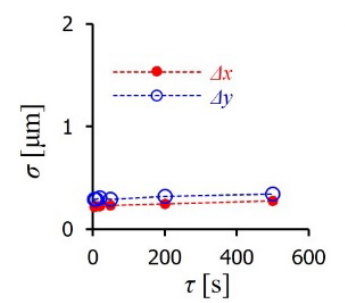

Stretching

(c)
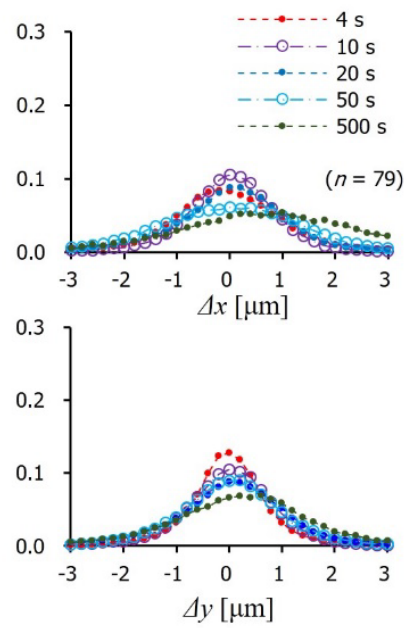

(g)

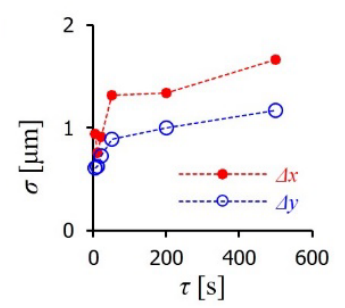

Freefall

(d)
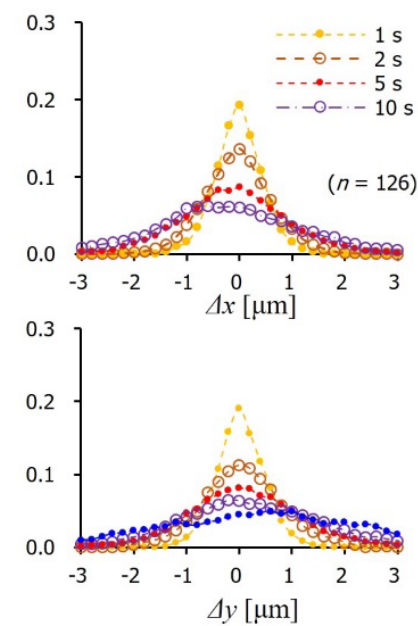

(h)

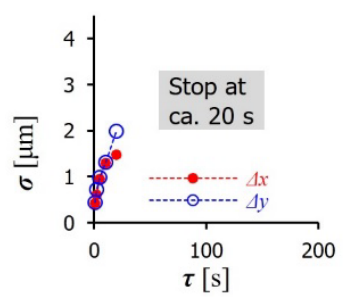

Figure 6. Distribution of particle displacements in intervals $\Delta t=1-500 \mathrm{~s}$. The particle trajectories in Figure 5 (a)-(d) were analysed. The probability of displacement is shown along the $\Delta x$ and $\Delta y$ directions. Distribution width $\sigma$ was estimated from Gaussian fitting and plotted for of each period $\tau$ in (e)-(h). For $\tau=500 \mathrm{~s}$ using the active matrix, Gaussian fitting was not performed because the distribution was almost flat. 
(a)

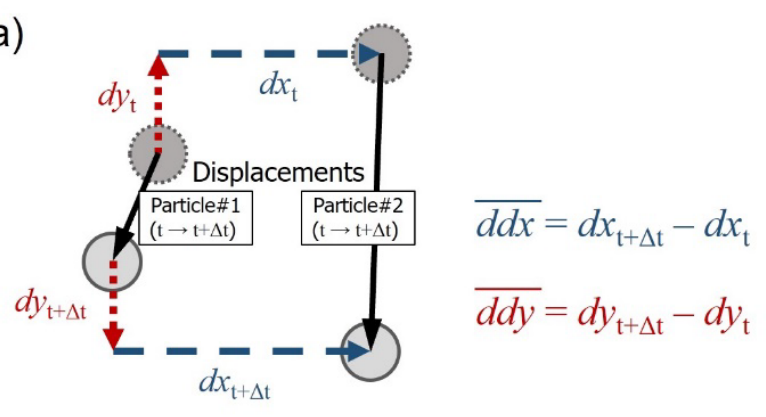

(d)

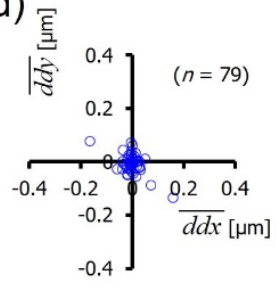

(e)

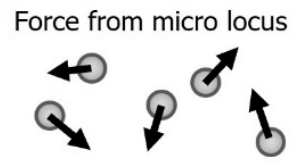

(f) Force from outer field

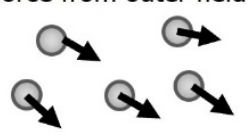

Figure 7. Comparison of relative displacements among particles. (a) Definition of relative displacements $(d d x, d d y)$. Relative displacements among particles fluctuating on active matrix of microtubule gel (b), on solid substrate decorated with microtubule gels and agitated by electric vibration motor (c), and on stretching elastic substrate STREX ${ }^{\circledR}(d)$. Diagram of likely movements of particles exposed to force from micro locus as active matrix (e) or force from outer field $(f)$.

which cannot be achieved by thermal fluctuation. ${ }^{58}$ The twodimensionally isotropic spread of interparticle fluctuation was efficiently generated by the active matrix [Figure 7(b)], whereas the spread remained one-dimensional for agitation driven by the external forces without the active matrix [Figures S4(d) and S4(h)]. The addition of a matrix without ATP further restricted the fluctuations [Figures 7(c) and 7(d)]. Two-dimensional interparticle fluctuations were qualitatively different from one-dimensional fluctuations and seemed to be generated by the forces from the micro locus [Figure 7(e)]. There, multiple collisions of microtubules in the active matrix driven by various kinesin molecules took place, leading to frequent switch-back turns to change the moving directions. In contrast, the particle movements often occur in the same direction under external forces [Figure 7(f)], and differences in the travel distance might cause one-dimensional interparticle fluctuations.

The frequent collisions of the active matrix which can produce zig-zag patterns in the particle trajectory [Figure 5(a)] may also be applicable for enhancing self-folding processes. ${ }^{62}$ In future work, other agitation methods driven by external forces such as magnetic fields or AC electric fields which do

not need chemical reactions may be combined to increase agitation control. ${ }^{63,64}$ In addition, the ability of the active matrix to move non-Brownian objects beyond the thermal fluctuation scale may contribute to perform hierarchical selfassembly based on precise design of building blocks, such as patchy colloids or Janus colloids. . $^{3,93,39,65-68}$ Their programmable self-assembly may allow an increase in the scale and elasticity tuning of the macrostructure under support of the bottom-up driving system of the active matrix. ${ }^{69-73}$ As the binding interaction of particles can be enhanced and destroyed depending on the temperature, especially for DNA affinity, moderate and controllable agitation is desirable also in the mesoscopic scale. ${ }^{69}$ Owing to the chemical energydependent mechanism, agitation by the active matrix is tuneable, and local control may be possible using lightresponding substrates such as caged-ATP. ${ }^{74}$ The tuneable agitation at the mesoscopic scale may also contribute to the study of active matters, including self-organisation consisting of reversible dynamic interactions of building blocks. ${ }^{75}$ The control of aggregation may even be applicable to living cells ${ }^{59}$ used as micro-objects because the active matrix can operate in living-cell compatible liquid. ${ }^{59}$ Thus, the enhancement of interparticle fluctuations by the active matrix can likely support a wide variety of applications, including those from biomedicine.

We characterise the driving properties of an active matrix consisting of crosslinked microtubules and kinesin. The stochastic conveyance of the microparticles on the active matrix enhances the isotropic spread of interparticle fluctuations among non-Brownian particles, which hardly travel under thermal fluctuation. The uniqueness of this agitation method is shown through comparisons with other agitation methods that use external forces from motor vibration or chamber stretching. As the interaction to move particles in the active matrix establishes a physical collision, this method can be generally applied to the fluctuation of large particles, including living cells, to enhance interparticle communication.

\section{Acknowledgements}

The plasmid construct of K560-GFP was generously provided by Prof. Ronald D. Vale and Prof. Michio Tomishige. This work was supported by the Leading Initiative for Excellent Young Researchers (LEADER) of MEXT and the Japan Society for the Promotion of Science (JSPS), by a grant from JSPS KAKENHI (No. T19K12775), by a JCG-S Scholarship Foundation research grant, and by a Takeda Science Foundation research grant. This work was also partially supported by a grant from JSPS KAKENHI (Nos. 16K12868 and 19KK0128) awarded to H. Y. Yoshikawa. 


\section{References}

1 Whitesides, G. M. \& Boncheva, M. Beyond molecules: Self-assembly of mesoscopic and macroscopic components. Proceedings of the National Academy of Sciences 99, 4769-4774, doi:10.1073/pnas.082065899 (2002).

2 Mastrangeli, M. et al. Self-assembly from milli- to nanoscales: methods and applications. Journal of Micromechanics and Microengineering 19, 083001, doi:10.1088/0960-1317/19/8/083001 (2009).

3 Buttinoni, I. et al. Dynamical Clustering and Phase Separation in Suspensions of Self-Propelled Colloidal Particles. Phys. Rev. Lett. 110, 5, doi:10.1103/PhysRevLett.110.238301 (2013).

4 Paulson, J. A., Mesbah, A., Zhu, X., Molaro, M. C. \& Braatz, R. D. Control of self-assembly in microand nano-scale systems. Journal of Process Control 27, 38-49, doi:10.1016/j.jprocont.2014.10.005 (2015).

5 Lash, M. H., Fedorchak, M. V., McCarthy, J. J. \& Little, S. R. Scaling up self-assembly: bottom-up approaches to macroscopic particle organization. Soft Matter 11, 5597-5609, doi:10.1039/C5SM00764J (2015).

Kamp, M. et al. Multivalent Patchy Colloids for Quantitative 3D Self-Assembly Studies. Langmuir 36, 2403-2418, doi:10.1021/acs.langmuir.9b03863 (2020).

Zhou, Y. et al. Reversible Janus particle assembly via responsive host-guest interactions. Chemical Communications $\mathbf{5 1}$, 2725-2727, doi:10.1039/c4cc09672j (2015).

Wang, L. K., Xia, L. H., Li, G., Ravaine, S. \& Zhao, X. S. Patterning the surface of colloidal microspheres and fabrication of nonspherical particles. Angew. Chem.-Int. Edit. 47, 4725-4728, doi:10.1002/anie.200801061 (2008).

Zhang, Z. \& Glotzer, S. C. Self-Assembly of Patchy Particles. Nano Letters 4, 1407-1413, doi:10.1021/n10493500 (2004).

Wilber, A. W. et al. Reversible self-assembly of patchy particles into monodisperse icosahedral clusters. The Journal of Chemical Physics 127, 085106, doi:10.1063/1.2759922 (2007).

1 Romano, F. \& Sciortino, F. Patchy from the bottom up. Nat. Mater. 10, 171-173, doi:10.1038/nmat2975 (2011).

2 Yi, G.-R., Pine, D. J. \& Sacanna, S. Recent progress on patchy colloids and their self-assembly. Journal of Physics: Condensed Matter 25, 193101, doi:10.1088/0953-8984/25/19/193101 (2013).

3 Glotzer, S. C. \& Solomon, M. J. Anisotropy of building blocks and their assembly into complex structures. Nat. Mater. 6, 557-562, doi:10.1038/nmat1949 (2007).

Sacanna, S., Pine, D. J. \& Yi, G.-R. Engineering shape: the novel geometries of colloidal self- assembly. Soft Matter 9, 8096-8106, doi:10.1039/C3SM50500F (2013).

Wang, Y. et al. Colloids with valence and specific directional bonding. Nature 491, 51-55, doi:10.1038/nature11564 (2012).

Chen, Q., Bae, S. C. \& Granick, S. Directed selfassembly of a colloidal kagome lattice. Nature 469, 381-384, doi:10.1038/nature09713 (2011).

Bowden, N., Terfort, A., Carbeck, J. \& Whitesides, G. M. Self-Assembly of Mesoscale Objects into Ordered Two-Dimensional Arrays. Science 276, 233-235, doi:10.1126/science.276.5310.233 (1997). Ashton, D. J., Jack, R. L. \& Wilding, N. B. Selfassembly of colloidal polymers via depletionmediated lock and key binding. Soft Matter 9, 96619666, doi:10.1039/C3SM51839F (2013).

Sacanna, S. et al. Shaping colloids for self-assembly. Nat Commun 4, 1688, doi:10.1038/ncomms2694 (2013).

Biancaniello, P. L., Kim, A. J. \& Crocker, J. C. Colloidal Interactions and Self-Assembly Using DNA Hybridization. Phys. Rev. Lett. 94, 058302, doi:10.1103/PhysRevLett.94.058302 (2005).

Valignat, M.-P., Theodoly, O., Crocker, J. C., Russel, W. B. \& Chaikin, P. M. Reversible self-assembly and directed assembly of DNA-linked micrometer-sized colloids. Proceedings of the National Academy of Sciences of the United States of America 102, 42254229, doi:10.1073/pnas.0500507102 (2005).

Nykypanchuk, D., Maye, M. M., van der Lelie, D. \& Gang, O. DNA-guided crystallization of colloidal nanoparticles. Nature 451, 549-552, doi:10.1038/nature06560 (2008).

23 Pawar, A. B. \& Kretzschmar, I. Fabrication, Assembly, and Application of Patchy Particles. Macromol Rapid Comm 31, 150-168, doi:10.1002/marc.200900614 (2010).

24 Jiang, X. C., Zeng, Q. H., Chen, C. Y. \& Yu, A. B. Self-assembly of particles: some thoughts and comments. Journal of Materials Chemistry 21, 16797-16805, doi:10.1039/c1jm12213d (2011). Di Michele, L. \& Eiser, E. Developments in understanding and controlling self assembly of DNA-functionalized colloids. Physical Chemistry Chemical Physics 15, 3115-3129, doi:10.1039/c3cp43841d (2013).

26 Di Michele, L. et al. Multistep kinetic self-assembly of DNA-coated colloids. Nat Commun 4, doi:10.1038/ncomms3007 (2013).

27 O'Brien, M. N., Jones, M. R. \& Mirkin, C. A. The nature and implications of uniformity in the hierarchical organization of nanomaterials. Proceedings of the National Academy of Sciences of the United States of America 113, 11717-11725, doi:10.1073/pnas.1605289113 (2016).

28 Li, W. Y. et al. Colloidal molecules and patchy particles: complementary concepts, synthesis and 
self-assembly. Chem. Soc. Rev. 49, 1955-1976, doi:10.1039/c9cs00804g (2020).

Bae, C., Kim, H., Moreno, J. M. M., Yi, G. R. \& Shin, H. Toward Coordinated Colloids: Site-Selective Growth of Titania on Patchy Silica Particles. Sci Rep 5, doi:10.1038/srep09339 (2015). Iwashita, Y. \& Kimura, Y. Spatial confinement governs orientational order in patchy particles. Sci Rep 6, doi:10.1038/srep27599 (2016).

31 Elwenspoek, M. et al. Self-assembly of (sub-)micron particles into supermaterials. Journal of Micromechanics and Microengineering 20, 064001, doi:10.1088/0960-1317/20/6/064001 (2010). Lash, M. H., Fedorchak, M. V., Little, S. R. \& McCarthy, J. J. Fabrication and Characterization of Non-Brownian Particle-Based Crystals. Langmuir 31, 898-905, doi:10.1021/la501511s (2015).

33 Choi, S.-W., Xie, J. \& Xia, Y. Chitosan-Based Inverse Opals: Three-Dimensional Scaffolds with Uniform Pore Structures for Cell Culture. Adv. Mater. 21, 2997-3001, doi:10.1002/adma.200803504 (2009).

34 Stachowiak, A. N., Bershteyn, A., Tzatzalos, E. \& Irvine, D. J. Bioactive Hydrogels with an Ordered Cellular Structure Combine Interconnected Macroporosity and Robust Mechanical Properties. Adv. Mater. 17, 399-403, doi:10.1002/adma.200400507 (2005).

35 Choi, S.-W., Cheong, I. W., Kim, J.-H. \& Xia, Y. Preparation of Uniform Microspheres Using a Simple Fluidic Device and Their Crystallization into Close-Packed Lattices. Small 5, 454-459, doi:10.1002/smll.200801498 (2009).

36 Grunwald, M., Tricard, S., Whitesides, G. M. \& Geissler, P. L. Exploiting non-equilibrium phase separation for self-assembly. Soft Matter 12, 15171524, doi:10.1039/c5sm01922b (2016).

37 Haghighat, B., Mastrangeli, M., Mermoud, G., Schill, F. \& Martinoli, A. Fluid-Mediated Stochastic SelfAssembly at Centimetric and Sub-Millimetric Scales: Design, Modeling, and Control. Micromachines 7, doi:10.3390/mi7080138 (2016).

38 Manoharan, V. N., Elsesser, M. T. \& Pine, D. J. Dense packing and symmetry in small clusters of microspheres. Science 301, 483-487, doi:10.1126/science.1086189 (2003).

39 Gong, Z., Hueckel, T., Yi, G.-R. \& Sacanna, S. Patchy particles made by colloidal fusion. Nature 550, 234-238, doi:10.1038/nature23901 (2017).

40 Ben Zion, M. Y. et al. Self-assembled threedimensional chiral colloidal architecture. Science 358, 633-+, doi:10.1126/science.aan5404 (2017).

41 Bechinger, C. et al. Active particles in complex and crowded environments. Reviews of Modern Physics 88, 045006, doi:10.1103/RevModPhys.88.045006 (2016).

42 Yang, Y. S., Barry, E., Dogic, Z. \& Hagan, M. F. Self-assembly of 2D membranes from mixtures of hard rods and depleting polymers. Soft Matter 8, 707714, doi:10.1039/c1sm06201h (2012).

Yan, J. et al. Reconfiguring active particles by electrostatic imbalance. Nat. Mater. 15, 1095-+, doi:10.1038/nmat4696 (2016).

44 Duguet, É., Hubert, C., Chomette, C., Perro, A. \& Ravaine, S. Patchy colloidal particles for programmed self-assembly. Comptes Rendus Chimie 19, 173-182, doi:10.1016/j.crci.2015.11.013 (2016). Erb, R. M., Son, H. S., Samanta, B., Rotello, V. M. \& Yellen, B. B. Magnetic assembly of colloidal superstructures with multipole symmetry. Nature 457, 999-1002, doi:10.1038/nature07766 (2009).

Yan, J., Bloom, M., Bae, S. C., Luijten, E. \& Granick, S. Linking synchronization to self-assembly using magnetic Janus colloids. Nature 491, 578-+, doi:10.1038/nature11619 (2012).

Han, M., Yan, J., Granick, S. \& Luijten, E. Effective temperature concept evaluated in an active colloid mixture. Proceedings of the National Academy of Sciences 114, 7513-7518, doi:10.1073/pnas.1706702114 (2017).

Maggi, C. et al. Self-Assembly of Micromachining Systems Powered by Janus Micromotors. Small 12, 446-451, doi:10.1002/smll.201502391 (2016).

49 Mallory, S. A. \& Cacciuto, A. Activity-assisted selfassembly of colloidal particles. Phys. Rev. E 94, doi:10.1103/PhysRevE.94.022607 (2016).

Mallory, S. A., Valeriani, C. \& Cacciuto, A. An Active Approach to Colloidal Self-Assembly. Annual Review of Physical Chemistry 69, 59-79, doi:10.1146/annurev-physchem-050317-021237 (2018).

51 Brangwynne , C. P., Koenderink , G. H., MacKintosh , F. C. \& Weitz , D. A. Cytoplasmic diffusion: molecular motors mix it up. Journal of Cell Biology 183, 583-587, doi:10.1083/jcb.200806149 (2008).

52 Kawamura, R., Kakugo, A., Shikinaka, K., Osada, Y. \& Gong, J. P. Ring-shaped assembly of microtubules shows preferential counterclockwise motion. Biomacromolecules 9, 2277-2282, doi:10.1021/bm800639w (2008).

53 Liu, H. Q. et al. Biomolecular Motor-Powered SelfAssembly of Dissipative Nanocomposite Rings. $A d v$. Mater. 20, 4476-4481, doi:10.1002/adma.200801291 (2008).

54 Ikuta, J. et al. Tug-of-war of microtubule filaments at the boundary of a kinesin- and dynein-patterned surface. Sci Rep 4, 8, doi:10.1038/srep05281 (2014). 55 Kaneko, T. et al. Transport of microtubules according to the number and spacing of kinesin motors on gold nano-pillars. Nanoscale 11, 98799887, doi:10.1039/C9NR01324E (2019).

56 Matsuda, K. et al. Artificial Smooth Muscle Model Composed of Hierarchically Ordered Microtubule Asters Mediated by DNA Origami Nanostructures. 
Nano Letters 19, 3933-3938, doi:10.1021/acs.nanolett.9b01201 (2019).

Sano, K. et al. Thermoresponsive microtubule hydrogel with high hierarchical structure. Biomacromolecules. 12, 1409-1413. doi: 1410.1021/bm101578x. Epub 102011 Mar 101530. (2011).

Kawamura, R., Sano, K. I., Ijiro, K. \& Osada, Y. Chemically cross-linked microtubule assembly shows enhanced dynamic motions on kinesins. RSC $A d v$. 4, 32953-32959, doi:10.1039/c4ra04491f (2014).

Kawamura, R., Uehara, D., Kobayashi, N., Nakabayashi, S. \& Yoshikawa, H. Y. Kinesin-Driven Active Substrate Giving Stochastic Mechanical Stimuli to Cells for Characterization. ACS Biomaterials Science \& Engineering 2, 2333-2338, doi:10.1021/acsbiomaterials.6b00538 (2016).

60 Castoldi, M. \& Popov, A. V. Purification of brain tubulin through two cycles of polymerizationdepolymerization in a high-molarity buffer. Protein Expr. Purif. 32, 83-88, doi:10.1016/s10465928(03)00218-3 (2003).

61 Case, R. B., Pierce, D. W., HomBooher, N., Hart, C. L. \& Vale, R. D. The directional preference of kinesin motors is specified by an element outside of the motor catalytic domain. Cell 90, 959-966, doi:10.1016/s0092-8674(00)80360-8 (1997).

62 Leong, T. G., Zarafshar, A. M. \& Gracias, D. H. Three-Dimensional Fabrication at Small Size Scales. Small 6, 792-806, doi:10.1002/smll.200901704 (2010).

63 Zhang, J., Luijten, E., Grzybowski, B. A. \& Granick, S. Active colloids with collective mobility status and research opportunities. Chem. Soc. Rev. 46, 55515569, doi:10.1039/c7cs00461c (2017).

64 Liljeström, V., Chen, C., Dommersnes, P., Fossum, J. O. \& Gröschel, A. H. Active structuring of colloids through field-driven self-assembly. Current Opinion in Colloid \& Interface Science 40, 25-41, doi:10.1016/j.cocis.2018.10.008 (2019).

65 Jones, M. R. \& Mirkin, C. A. Self-assembly gets new direction. Nature 491, 42-43, doi:10.1038/491042a (2012).

66 Gröschel, A. H. et al. Guided hierarchical coassembly of soft patchy nanoparticles. Nature $\mathbf{5 0 3}$, 247-251, doi:10.1038/nature12610 (2013).

67 Lunn, D. J., Finnegan, J. R. \& Manners, I. Selfassembly of "patchy" nanoparticles: a versatile approach to functional hierarchical materials. Chemical Science 6, 3663-3673, doi:10.1039/c5sc01141h (2015).

68 Choueiri, R. M. et al. Surface patterning of nanoparticles with polymer patches. Nature 538, 7983, doi:10.1038/nature19089 (2016).

69 Kim, A. J., Biancaniello, P. L. \& Crocker, J. C. Engineering DNA-mediated colloidal crystallization.
Langmuir 22, 1991-2001, doi:10.1021/la0528955 (2006).

70 Rogers, W. B., Shih, W. M. \& Manoharan, V. N. Using DNA to program the self-assembly of colloidal nanoparticles and microparticles. Nature Reviews Materials 1, 16008, doi:10.1038/natrevmats.2016.8 (2016).

Ong, L. L. et al. Programmable self-assembly of three-dimensional nanostructures from 10,000 unique components. Nature 552, 72-+, doi:10.1038/nature24648 (2017).

72 Chakraborty, I., Meester, V., van der Wel, C. \& Kraft, D. J. Colloidal joints with designed motion range and tunable joint flexibility. Nanoscale 9, 7814-7821, doi:10.1039/c6nr08069c (2017).

73 Morphew, D., Shaw, J., Avins, C. \& Chakrabarti, D. Programming Hierarchical Self-Assembly of Patchy Particles into Colloidal Crystals via Colloidal Molecules. ACS Nano 12, 2355-2364, doi:10.1021/acsnano.7b07633 (2018).

74 Tucker, R., Katira, P. \& Hess, H. Herding Nanotransporters: Localized Activation via Release and Sequestration of Control Molecules. Nano Letters 8, 221-226, doi:10.1021/n1072516n (2008).

75 Lehn, J.-M. Perspectives in Chemistry-Steps towards Complex Matter. Angewandte Chemie International Edition 52, 2836-2850, doi:10.1002/anie.201208397 (2013). 